\section{Protocolo de carga inmediata so- bre implantes en mandíbula edéntula}

Castilho AA, Bambace AMJ, Saavedra GSFA, Ccahuana-Vásquez VZ, Kimpara ET. Protocolo de carga inmediata sobre implantes en mandíbula edéntula. Rev Estomatol Herediana. 2007; 17(2):84-89.

\section{RESUMEN}

El protocolo de carga convencional iniciada por Brånermark en dos fases quirúrgicas ha sido evaluado durante los últimos 30 años bajo todas las condiciones clínicas posibles. Este tratamiento ha resuelto problemas relacionados a la retención y estabilidad de prótesis totales removibles convencionales, que perjudican función, la estética, la salud psicológica y las relaciones sociales de sus usuarios. Sin embargo, estas rehabilitaciones normalmente involucran tratamientos largos, con dos intervenciones quirúrgicas y varios ajustes de la prótesis provisional, siendo repetitivo para el profesional y el paciente. En comparación, la carga inmediata es un protocolo relativamente nuevo que necesita de una estricta evaluación clínica. El protocolo de carga inmediata sobre implantes es una de las opciones preferidas por los pacientes, pues consiste en la colocación de los implantes e inmediata instalación de la prótesis total fija, siendo mas rápida y menos traumática. Sin embargo, el éxito de la carga inmediata depende de algunos pre-requisitos importantes las condiciones del paciente, así como la destreza y el conocimiento técnico del implantologo y el rehabilitador oral. Este trabajo describe el éxito de un caso clínico de protocolo con carga inmediata sobre implantes, en que sea relatado los procedimientos clínicos y de laboratorio con un seguimiento durante 12 meses. Debido a sus ventajas, creemos que esta puede ser una técnica utilizada y previsible cuando bien indicada y ejecutada.

Palabras clave: IMPLANTES DENTALES / PRÓTESIS DENTAL DE SOPORTE IMPLANTADO / DENTADURA COMPLETA INFERIOR.

Immediate loading protocol of implants in edentulous mandible.

ABSTRACT

The protocol for conventional loading started by Brånermark in two surgical stages has been evaluated over the last 30 years under all the possible clinical conditions. This treatment has solved problems related to retention and stability of conventional removable total prostheses, which damage function, aesthetics, psychological health and user's social relationships. By comparison, immediate loading is a relatively new protocol that requires strict clinical evaluation. The immediate loading protocol over implants is the option of choice for patients, since it consist on the placement of implants followed by the immediate installation of the fixed prosthesis, which is faster and less traumatic. However, the success of the immediate load depends on some prerequisites that are inherent to patients condition as well as to the professional skills. This article describes the success of a clinical protocol with immediate loading over implants, in which clinical and laboratory procedures are reported with a one year follow-up. Due to its advantages, we believe that this is a useful and predictable technique, if correctly used and executed.

Key words: DENTAL IMPLANTS / IMPLANT-SUPPORTED DENTAL PROSTHESIS / COMPLETE LOWER DENTURE.
Anderson Almeida Castilho'

Andréa Moreira Jacobucci

Bamabace $^{1}$

Guilherme de Siqueira Ferreira

Anzaloni Saavedra ${ }^{2}$

Vanessa Zulema Ccahuana

Vásquez $^{3}$

\section{Estevão Tomomitsu Kimpara ${ }^{4}$}

${ }^{1}$ Cirujano Dentista.

${ }^{2}$ Alumno del Curso de Doctorado en Odontología

Restauradora. Especialidad Prótesis Dental.

Universidad Estadual Paulista Júlio Mesquita Filho /

Facultad de Odontología - Campus de São José dos

Campos, UNESP, São Paulo, Brasil.

Campos, UNESP, São Paulo, Brasil.

Estomatológica. Facultad de Estomatología.

Universidad Peruana Cayetano Heredia.

${ }^{4}$ Docente del Departamento de Materiales

Odontológicos y Prótesis. Universidad Estadual

Paulista Júlio Mesquita Filho / Facultad de

Odontología - Campus de São José dos Campos,

UNESP, São Paulo, Brasil.

\section{Correspondencia}

Vanessa Zulema Ccahuana Vásquez

Carlos de los Heros 213, Lima 21 - Perú.

Teléfono: 4609463

e-mail:vanesca@dr.com

Recibido :21 de marzo del 2007

Aceptado : 15 de julio del 2007

\section{Introducción}

De acuerdo con el protocolo de Brånermark, el periodo inicial en que los implantes deben permanecer sin cualquier tipo de presión es muy importante para que ocurra la oseointegración entre el hueso y el implante, sin embargo estudios recientes presentan la colocación de implantes con carga inmediata en mandíbulas edéntulas (1).

Tradicionalmente, el protocolo de utilización de implantes oseointegrables envuelve dos fases quirúrgicas y un periodo prolongado de cicatrización durante el cual cargas funcionales deben ser evitados (2-7). De esta manera la rehabilitación oral de los pacientes era realizada solamente después del periodo de oseo-integración de los implantes colocados, siendo entre tres e seis meses, dependiendo de la calidad del tejido óseo $(8,9)$. Sin embargo diferentes técnicas han sido propuestas con la finalidad de resumir el tiempo entre la instalación del implante y la colocación de la prótesis. El protocolo quirúrgico de un solo paso involucra la aplicación de carga inmediata a través de la colocación de una prótesis provisional sobre los implantes recién colocados, representando una alternativa viable para la rehabilitación de pacientes con perdi- da parcial o total de dientes. Schnitman et al., relataron el primer trabajo sobre carga inmediata en implantes, concluyendo que la activación precoz no afecta el pronostico del tratamiento (10). Asimismo, otros reportes de la literatura demuestran que cuando respetadas determinadas condiciones, la carga inmediata puede constituir una alternativa de buena previsibilidad en comparación al protocolo tradicional de dos fases quirúrgicas (11-13).

De forma general, los trabajos de carga inmediata descritos en la literatura enfatizan que la ferulización rígida de los implantes a través de una 
infraestructura metálica es condición necesaria para la viabilidad de este procedimiento (14). Esta condición exige procedimientos clínicos y de laboratorio que demandan la finalización del caso en 48 ó 72 horas después de la cirugía. Brånermark et al. (15), introdujeron un sistema que reducía el tiempo de cicatrización sin carga, Brånermark Novum, desarrollando un protocolo de tratamiento de un día.

En este reporte de caso clínico se presenta la rehabilitación con carga inmediata de una mandíbula edéntula a través de una prótesis total fija implanto soportada.

\section{Caso}

Paciente de sexo femenino de 49 años portadora de una prótesis total inferior, se presentó en la clínica de especialización en Implantologia APCD-SBC, SP con la queja de insatisfacción debido a la falta de inestabilidad y retención de la prótesis dental. Al examen clínico intraoral se observó la reabsorción ósea alveolar con reborde residual mandibular, ofreciendo limitadas condiciones de retención y estabilidad para una prótesis total convencional. Al examen radiográfico mostró reabsorción ósea moderada en la región posterior de mandíbula. Sin embargo, en la región interforamen fue posible identificar altura ósea y perímetro de arco suficientes para la instalación de cinco implantes (Fig. 1). Después de esta evaluación clínica y radiográfica, además de una cuidadosa anamnesis y exámenes laboratoriales así como el análisis de los modelos articulados, fue propuesta la confección de una prótesis fija inferior sobre implantes a través del protocolo de carga inmediata.

Fueron confeccionadas nuevas prótesis totales, respetando la secuencia clínica de impresión anatómica y funcional, registro intermaxilar con rodetes de orientación en cera, prue- ba de dientes y acrilización. Con estas nuevas prótesis se restableció la dimensión vertical de oclusión del paciente restaurando mejor la función y la estética. Se realizó un desgaste en la prótesis total inferior en el área lingual delimitada entre primeras premolares que sirvió para la orientación en la posición de los implantes y el acto de transferencia de los mismos a un modelo de trabajo .

El acto quirúrgico se inicio con la incisión a la altura de la cresta ósea desde el área posterior a los forámenes mentonianos con colgajo mucoperiostico y exposición de los paquetes vasculo nerviosos mentonianos (Fig. 2). Se inició la colocación de los implantes dentro de los límites vestibular y lingual, verificando las inclinaciones e dirección con pines guías. Así, fueron colocados 5 implantes cónicos Ace de Bortoli de conexión interna con dimensiones de 3,75 x $13 \mathrm{~mm}$, siendo que todos tuvieron una trabamiento inicial superior a los $40 \mathrm{~N}$, lo que garantizó la indicación de la carga inmediata en este caso. Los pilares intermediarios tipo Esteticone Ace de Bortoli para hexano interno

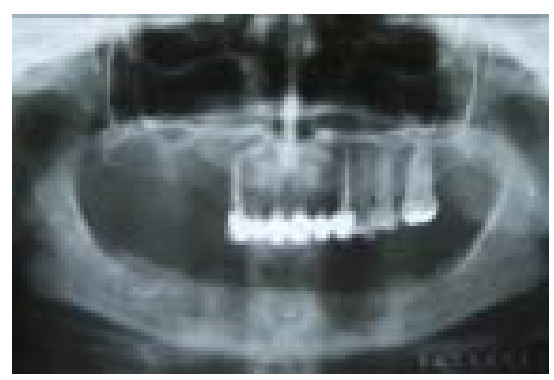

Fig. 1. Radiografia panorámica preoperatoria.

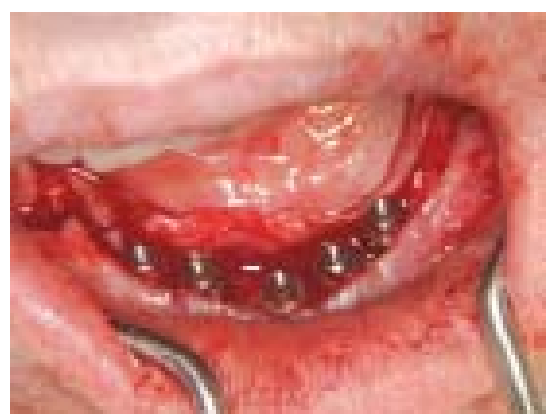

Fig. 3. Colocación de pilares esteticone.
Microunit con cintas metálicas de 3mm fueron instalados y recibieron un torque de $20 \mathrm{~N}$ (Fig. 3). El procedimiento quirúrgico fue finalizado con la sutura del colgajo.

Los procedimientos protésicos se iniciaron con la prueba de los cilindros fundidos previamente confeccionados a partir de una infraestructura diseñada en base a los modelos de estudio, una vez verificado el asentamiento de los cilindros en cada pilar, se procedió a realizar la ferulización de estos con acrílico de combustión rápida, resina GC Pattern, Tokio, Japón (Fig 4). Inmediatamente se posicionó la prótesis inferior con el recorte en lingual verificando la estabilidad y espacio suficiente con respecto a los cilindros posicionados, siendo registrada nuevamente la dimensión vertical oclusal con las dos prótesis en posición. Este procedimiento se justificó para evitar alteraciones en la Dimensión Vertical Oclusal (DVO) cuando capturados los cilindros por medio de acrílico. Una vez verificado esta posición se procedió a capturar los cilindros aun

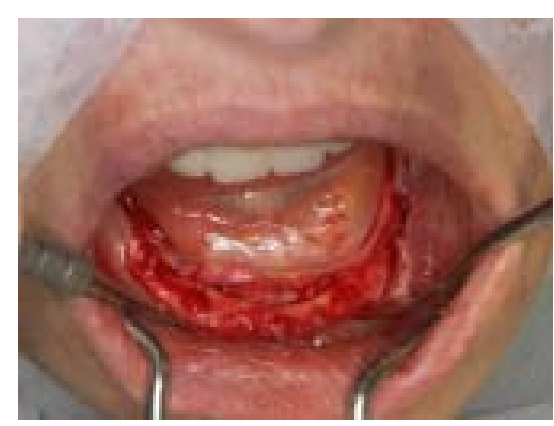

Fig. 2. Colgajo mucoperiósteo, exposición del área receptora de implantes.

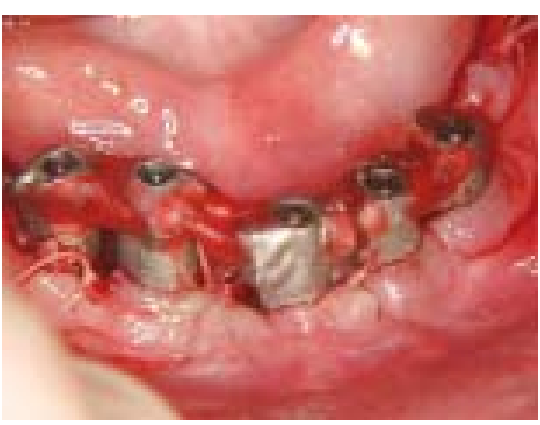

Fig. 4. Unión de cilindros colados con resina acrílica de combustión rápida. 
posicionados en boca con la prótesis usando acrílico GC Pattern (Fig. 5), después de colocado el acrílico se solicitó al paciente ocluir y mantenerse en esa posición mientras verificábamos la DVO, con las marcas obtenidas anteriormente con el compás de Willis y el método de la plenitud facial. Los accesos para los tornillos protésicos fueron liberados teniendo cuidado de no comprometer las superficies oclusales de los dientes posteriores. Polimerizado el acrílico, el conjunto prótesis y cilindros es removido, obteniendo un index bastante fiel de la localización de los implantes (Fig. 6). El paciente recibió tapas de protección Ace de Bortoli sobre los pilares mientras aguardaba los procedimientos de laboratorio.

Con los análogos de los implantes obtuvimos un modelo de trabajo que facilitó el procedimiento de soldadura de la infraestructura teniendo cuidado en todo momento de respetar el asentamiento pasivo de esta. Una vez realizada la prueba en boca de la infraestructura (Fig. 7), se envió al laboratorio para realizar el acrilizado fi-

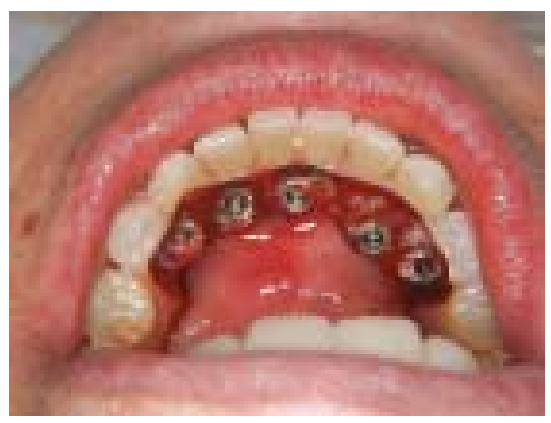

Fig. 5. Unión con acrílico de la prótesis total inferior con los cilindros.

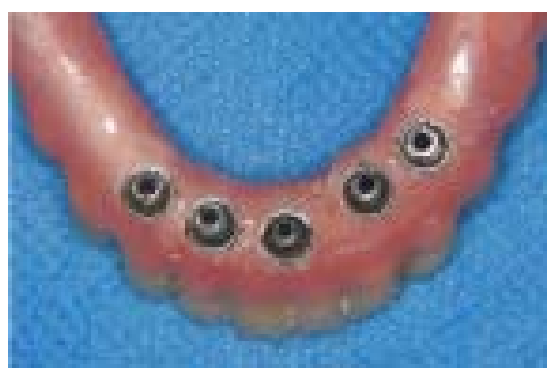

Fig. 8. Vista de prótesis acrilizada y pulida respetando el acceso a los cilindros. nal, siendo realizado por un incremento gradual de acrílico rosado químicamente activado, de forma de minimizar alteraciones dimensionales, la polimerización final fue realizada con olla a presión para acrilizado. Toda la extensión de la prótesis a partir de distal de la primera molar eliminada. Los espacios para la higiene fueron cuidadosamente esculpidos entre las bases de los cilindros. En el sentido vestíbulo lingual de la prótesis fue arrendondada siendo realizado el formato de póntico en bala. Después del acabado, la prótesis fue pulida y el procedimiento de laboratorio culminado (Fig. 8).

Removidas las tapas de protección, la prótesis fue colocada en posición para evaluación de su adaptación (Fig. 9). Estando en condiciones adecuadas, fue instalada empleando los tornillos de titanio realizando el ajuste con torquímetro manual de 10 N. Los orificios de acceso fueron cubierto con gutapercha y resina fotopolimerizable. La oclusión fue verificada teniendo el cuidado de garantizar apenas contactos céntricos en los dientes posterio-

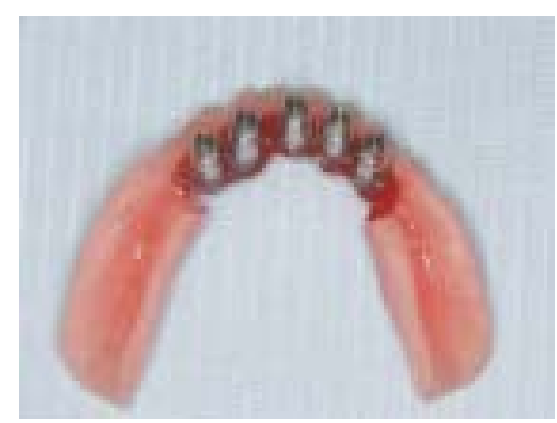

Fig. 6. Index

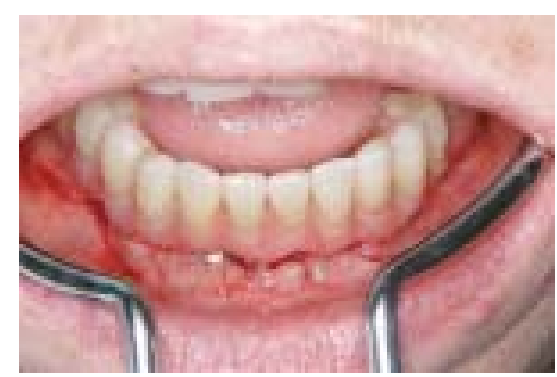

Fig. 9. Prueba de la prótesis híbrida sobre los pilares esteticones. res. El paciente fue orientado con indicaciones detalladas respecto a la alimentación, higiene y medicación, finalizando el procedimiento quirúrgico, protésico y laboratorial en 8 horas. En los controles clínicos posteriores se observó discreto edema e ausencia de dolor, infección o parestesia. Además en tomas radiográficas posteriores fue posible constatar una correcta disposición de los implantes en relación a las estructuras adyacentes y la perfecta adaptación de los componentes de la prótesis sobre los pilares (Fig. 10).

\section{Discusión}

La rehabilitación de arcos edéntulos empleando implantes oseointegrados como anclaje para prótesis a partir del protocolo tradicional de dos estadios quirúrgicos tienen reportes en la literatura con resultados clínicos satisfactorios $(1,15)$. Según Albrektsson (4), el protocolo tradicional establece la necesidad de mantener los implantes sin fuerzas oclusales incidiendo sobre ellos, esperando un periodo de seis meses y cuatro

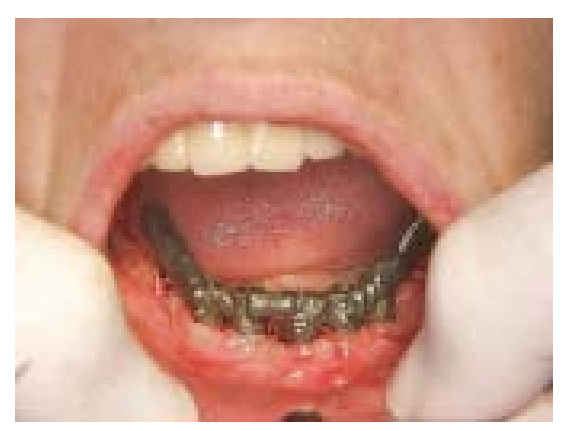

Fig. 7. Prueba en boca de estructura metálica fundida sobre pilares esteticones.

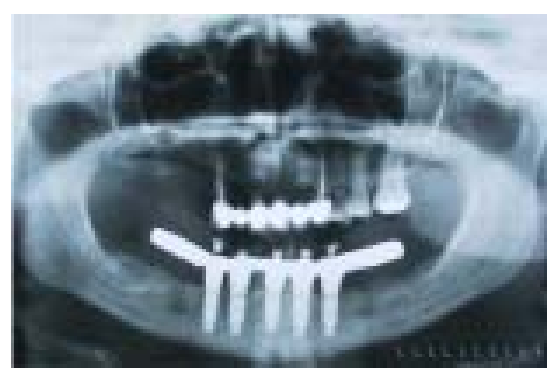

Fig. 10. Radiografia panorámica postratamiento. 
ses para el maxilar superior e inferior respectivamente (16). Durante este periodo de espera para que ocurra la oseointegración, los pacientes deben usar prótesis removibles o dispensar el uso de ellas. El uso de estas prótesis temporales en la mayoría de situaciones con pobre estabilidad y retención generan situaciones de incomodidad, necesidad de ajustes frecuentes eventuales problemas de convivió psicológico y social (17).

En uno de los primeros reportes de carga inmediata, Schnitman (10) realizó un estudio donde colocó entre 5 a 6 implantes en mandíbulas edéntulas, e instaló posteriormente una prótesis total fija sobre apenas tres pilares protésicos, con el objetivo de comparar implantes cargados de forma inmediata en la mandíbula, frente a implantes sumergidos en el mismo paciente que utilizaba de control. Los autores no encontraron diferencias estadísticamente significativas cuando compararon los niveles de falla de implantes, inmediatamente cargados con los implantes sin carga, en el mismo paciente, durante un periodo de 5 años. Después de 10 años de seguimiento Schnitman et al., (17) reportaron un índice de éxito de 93,4\% para todos los implantes, $84,7 \%$ para los implantes con carga inmediata y de $100 \%$ para los implantes sumergidos. Los resultados mostraron que a pesar del éxito a corto plazo en implantes mandibulares con función inmediata, los resultados a largo plazo deben ser cuidadosamente considerados, principalmente para los implantes colocados en áreas mas distales.

Para el éxito de este protocolo con carga inmediata, algunas condiciones deben ser respetadas. Este protocolo debe ser aplicado en pacientes edéntulos con buena salud, no fumantes, ausencia de bruxismo, portadores de hueso tipo I o II y moderada reabsorción ósea, capaz de recibir como mínimo 4 implantes en el área delimitada entre forámenes mentonianos $(17,18)$. La estabilidad primaria de los implantes es otro factor importante pues deben presentar un torque de incersión superior a los 40N y uso de implantes con $10 \mathrm{~mm}$ de longitud como mínimo, debiendo estar rodeado por al menos $1 \mathrm{~mm}$ de hueso en sentido bucolingual (19). Según Brunski (19) el proceso de oseointegración depende de la ausencia de micromovimientos en la interfase implante con el tejido óseo durante el periodo de cicatrización inicial, pues movimientos superiores a $100 \mu$ m para implantes con superficie lisa y $150 \mu \mathrm{m}$ para implantes rugosos generan la formación de tejido fibroso o fibrocartilaginoso interfacial en vez de tejido óseo. En 1993, Brunski (20) reafirma que la carga inmediata puede ser realizada desde que no se produzca micromovimientos en los procedimientos ejecutados. La disminución del micromovimiento durante el periodo de remodelación ósea puede lograrse con una ferulización rígida e inmediata de todos los implantes, a través de una prótesis provisional atornillada con un perfecto ajuste pasivo (21).

Diferentes técnicas que buscan reducir el tiempo para la finalización de trabajos fueron publicados. En 1999, Brånemark (15) presentó el Novum System (Brånemark Novum, Nobel Biocare, Göteborg, Suecia) para carga inmediata. El sistema une tres implantes posicionados en la región anterior de la mandíbula, de 4mm de diámetro y longitud mínima de 13mm. Estos implantes son conectados a través de una plataforma de titanio prefabricada. Sobre ésta se atornilla una supra-estructura que porta la prótesis fija. Así, la finalización de un caso era posible en un día. Concluyeron después de un seguimiento de tres años que esos tres implantes eran suficien- tes para soportar usa prótesis híbrida.

Wolfinger en 2003 (22), en un estudio de 3 a 5 años de seguimiento con implantes Brånemark configuró dos grupos de tratamiento. El primer grupo era cargado con 6 implantes tanto en el sector anterior como posterior de la mandíbula. El segundo grupo era tratado con 10 implantes por mandíbula, cargando 4 de ellos con una prótesis provisional acrílica y dejando los 6 restantes sumergidos para descubrirlos en un segundo procedimiento. La supervivencia de los implantes cargados de forma inmediata del primer brazo del estudio fue del $97 \%$, mientras que del segundo fue del $80 \%$. Este estudio evidencia la ineficiencia de sumergir implantes de rescate protésico en vez de dar más estabilidad a la prótesis provisional con un número adecuado de ellos.

Maló en 2003 (13), presentó un estudio retrospectivo posicionando $4 \mathrm{im}$ plantes Brånemark interforaminales en 44 pacientes. Demostró la eficacia del uso de implantes angulados a $45^{\circ}$ colocados próximos al foramen mentoniano para obtener una posición más distal de la superestructura sin comprometer el nervio alveolar inferior. La tasa acumulativa de supervivencia con un seguimiento de 1 a 3 años fue del $96,7 \%$ y la supervivencia de la prótesis del $100 \%$.

Testori en 2004 (23), publicó el primer estudio prospectivo multicéntrico en implantes Osseotite (3i, Implant Inovations, EE.UU) sobre carga inmediata mandibular de 62 pacientes con cinco implantes interforaminales para soportar una prótesis híbrida provisional con extensiones en oclusión completa. Mostró una tasa acumulativa de supervivencia del 99,4\% a los dos años, sin cambios significativos en el hueso marginal.

El desarrollo eficiente y sin demora del tiempo quirúrgico con un pre- 
meoperatorio bien planeado, incluyendo una detallada historia clínica, estudio de imágenes, guía quirúrgica y encerado diagnóstico, ofrecen una seguridad muy grande en la instalación de los implantes. Debido al avance de investigaciones científicas, muchas técnicas se han desarrollado, facultando así al implantólogo de utilizar la carga inmediata en su rutina de trabajo como elemento rehabilitador.

\section{Conclusiones}

El éxito de la carga inmediata esta relacionadas con características individuales de cada paciente y con características de la rehabilitación implanto-soportada a ser realizada. Factores como la salud general, cualidad ósea, potencial de cicatrización e individualidad genética son importantes para la ejecución de este procedimiento. Así mismo, la región seleccionada, el trabamiento inicial de los implantes, el tipo de prótesis que será realizada sobre los implantes y su relación con la arcada antagonista también son considerados para el uso de la carga inmediata. A pesar de las ventajas de un único tiempo quirúrgico, de un periodo de tratamiento relativamente menor, de mayores beneficios psicológico y sociales es importante no generalizar esta opción de tratamiento para los pacientes, el proceso de selección del paciente, el estudio detallado del caso y dominio de la técnica aún siguen siendo los factores decisivos para la ejecución de este protocolo de carga inmediata.

\section{Referencias Bibliográficas}

1. 1. Lorenzoni M, Pertl C, Zhang $\mathrm{K}$, Wegscheider WA. In-patient comparison of immediately loaded and non-loaded implants within 6 months. Clin Oral Implants Res. 2003; 14(3):2739.

2. Branemark PI, Hansson BO,
Adell R, Breine U, Lindstrom J, Hallen O, Ohman A. Osseointegrated implants in the treatment of the edentulous jaw. Experience from a 10-year period. Scand J Plast Reconstr Surg Suppl. 1977; 16:1-132.

3. Adell R, Lekholm U, Rockler B, Branemark PI. A 15-year study of osseointegrated implants in the treatment of the edentulous jaw. Int J Oral Surg. 1981; 10(6):387416.

4. Albrektsson T, Branemark PI, Hansson HA, Lindstrom J. Osseointegrated titanium implants. Requirements for ensuring a long-lasting, direct bone-to-implant anchorage in man. Acta Orthop Scand. 1981; 52(2):155-70.

5. Skalak R. Biomechanical considerations in osseointegrated prostheses. J Prosthet Dent. 1983; 49(6):843-8.

6. Zarb GA, Jansson $\mathrm{T}$. Prosthodontic procedures. In: Brånemark PI. Tissue integrated prostheses. Chicago: Quintessence: 1985. p. 241-82.

7. Albrektsson $\mathrm{T}$, Jansson $\mathrm{T}$, Lekholm U. Osseointegrated dental implants. Dent Clin North Am. 1986; 30(1):151-74.

8. Lazzara RJ, Porter SS, Testori T, Galante J, Zetterqvist L. A prospective multicenter study evaluating loading of osseotite implants two months after placement: one-year results. J Esthet Dent. 1998; 10(6):280-9.

9. Bezerra F, Sousa SW, Duarte LR, Regis MB. Protocolo de carga inmediata funcional: Relato de casos clínicos. Inovations J. 2003; 7(1):6-13.

10.Schnitman PA, Wohrle PS, Rubenstein JE. Immediate fixed interim prostheses supported by two-stage threaded implants: methodology and results. J Oral Implantol. 1990; 16(2):96-105.

11. Salama H, Rose LF, Salama M, Betts NJ. Immediate loading of bilaterally splinted titanium rootform implants in fixed prosthodontics--a technique reexamined: two case reports. Int J Periodontics Restorative Dent. 1995;15(4):344-61.

12.Balshi SF, Wolfinger GJ, Balshi TJ. A prospective study of immediate functional loading, following the Teeth in a Day protocol: a case series of 55 consecutive edentulous maxillas. Clin Implant Dent Relat Res. 2005; 7(1):24-31.

13. Malo P, Rangert B, Nobre M. "All-on-Four" immediate-function concept with Branemark System implants for completely edentulous mandibles: a retrospective clinical study. Clin Implant Dent Relat Res. 2003; 5(Suppl 1):2-9.

14. Tarnow DP, Emtiaz S, Classi A. Immediate loading of threaded implants at stage 1 surgery in edentulous arches: ten consecutive case reports with 1to 5-year data. Int J Oral Maxillofac Implants. 1997; 12(3):319-24.

15. Branemark PI, Engstrand P, Ohrnell LO, Grondahl K, Nilsson P, Hagberg K, Darle C, Lekholm U. Branemark Novum: a new treatment concept for rehabilitation of the edentulous mandible. Preliminary results from a prospective clinical follow-up study. Clin Implant Dent Relat Res. 1999; 1(1):2-16. 16. Lekholm U. Clinical procedures for treatment with osseointegrated dental implants. J Prosthet Dent. 1983; 50(1):11620.

17. Schnitman PA, Wohrle PS, 
Rubenstein JE, DaSilva JD, Wang NH. Ten-year results for Branemark implants immediately loaded with fixed prostheses at implant placement. Int J Oral Maxillofac Implants. 1997; 12(4):495-503.

18. Babbush CA, Kent JN, Misiek DJ. Titanium plasma-sprayed (TPS) screw implants for the reconstruction of the edentulous mandible. J Oral Maxillofac Surg. 1986; 44(4):274-82.

19. Brunski JB. In vivo bone response to biomechanical loading at the bone/dental- implant interface. Adv Dent Res. 1999; 13:99-119.

20.Brunski JB. Avoid pitfalls of overloading and micromotion of intraosseous implants. Dent Implantol Update. 1993; 4(10):77-81.

21. Horiuchi K, Uchida H, Yamamoto $\mathrm{K}$, Sugimura M. Immediate loading of Branemark system implants following placement in edentulous patients: a clinical report. Int J Oral Maxillofac Implants. 2000; 15(6):824-30.

22. Wolfinger GJ, Balshi TJ, Rangert

B. Immediate functional loading of Branemark system implants in edentulous mandibles: clinical report of the results of developmental and simplified protocols. Int J Oral Maxillofac Implants. 2003; 18(2):250-7.

23. Testori T, Del Fabbro M, Galli F, Francetti L, Taschieri S, Weinstein R. Immediate occlusal loading the same day or the after implant placement: comparison of 2 different time frames in total edentulous lower jaws. J Oral Implantol. 2004; 30(5):307-13. 